\title{
Cecal MicroRNAome response to Salmonella enterica serovar Enteritidis infection in White Leghorn Layer
}

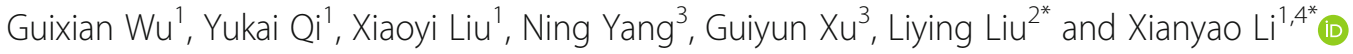

\begin{abstract}
Background: Salmonella enterica serovar Enteritidis (SE) is a food-borne pathogen and of great threat to human health through consuming the contaminated poultry products. MicroRNAs (miRNAs) play an important role in different biological activities and have been shown to regulate the innate immunity in the bacterial infection. The objective of this study is to identify miRNAs associated with SE infection in laying chicken cecum.

Results: Average number of reads of three libraries constructed from infected and non-infected chickens was 12,476,156 and 10,866,976, respectively. There were 598 miRNAs including 194 potential novel miRNAs identified in which 37 miRNAs were significantly differentially expressed between infected and non-infected chickens. In total, 2897 unique target genes regulated by differentially expressed miRNAs were predicted, in which, 841 genes were uniquely regulated by up-regulated miRNAs (G1), 636 genes were uniquely regulated by down-regulated miRNAs (G2), and 1420 were co-regulated by both up and down- regulated miRNAs (G3). There were 118, 73 and 178 GO (Gene ontology) BP (Biological process) terms significantly enriched in G1, G2 and G3 groups, respectively. More immune-related GO BP terms than metabolism-related terms were found in G1. Expression of 12 immune-related genes of four differentially expressed miRNAs was detected through qRT-PCR. The regulatory direction of gga-miR1416-5p, gga-miR-1662, and gga-miR-34a-5p were opposite with the target genes of TLR21, BCL10, TLR1LA, NOTCH2 and THBS1, respectively.

Conclusion: The miRNAs contribute to the response to SE infection at the onset of egg laying through regulating the homeostasis between metabolism and immunity. The gga-miR-125b-5p, gga-miR-34a-5p, gga-miR-1416-5p and gga-miR-1662 could play an important role in SE infection through regulating their target genes. The finding herein will pave the foundation for the studies of microRNA regulation in SE infection in laying hens.
\end{abstract}

Keywords: Laying chicken, miRNA, Salmonella enterica serovar Enteritidis, Next generation sequencing

\section{Background}

Salmonellosis is one of the most important food-borne diseases, with an estimated one million human cases and more than 350 deaths occurring each year in the United States [1]. Non-typhoidal Salmonella is the main cause of foodborne disease in the United States [2]. Salmonella enterica serotype Enteritidis (SE) is one of the most common serotypes of Salmonella bacteria reported worldwide

\footnotetext{
* Correspondence: Iyliu@sdau.edu.cn; xyli@sdau.edu.cn

${ }^{2}$ College of Life Science, Shandong Agricultural University, Tai'an, Shandong 271018, China

${ }^{1}$ College of Animal Science and Technology, Shandong Agricultural

University, Tai'an, Shandong 271018, China

Full list of author information is available at the end of the article
}

which is the major source of human intestinal infections [3]. The animal or animal product including poultry, eggs, milk and milk products could be the carriers of SE which threaten the human health [4-6]. Salmonella organisms could penetrate the intestines mucosal epithelium to outcompete the resident microbial flora and cause the pathological reaction [7]. Egg-related salmonellosis is costing \$44 million per year in Australia [8].

MicroRNAs (miRNAs) are small (19 24 nt long), noncoding, single-stranded and conserved RNAs. It performs multiple functions through regulating gene expression mainly at the post-transcriptional levels [9]. It plays an important role in different biological activities such as the development, cell differentiation and disease $[10,11]$. MiRNA 
could regulate innate immunity caused by viruses, bacteria, fungi, and protozoa infection [12, 13]. MiR-21, miR-146a/b and miR-155 were obviously up-regulated in rat's mononuclear cells after Salmonella infection [14, 15]. Let-7 was down-regulated to induce the release of cytokine IL6 (interleukin 6) and IL10 to participate in the regulation of immune response to Salmonella infection in macrophages [14]. MiRNAs also could buffer and alter the variance of relatively lowly expressed genes in the response to Salmonella infection in pig [16]. However, the responsive miRNAs in laying hen Salmonella infection is still unclear.

The objective of the present study is to discover the miRNAs in the response to Salmonella infection in chicken cecum at the onset of egg laying. Next generation sequencing (NGS) has been widely used to analyze the miRNA expression profile in many studies [17-19]. In the current study, the Solexa Sequencing method was used to detect differentially expressed miRNAs in the response to SE infection in White Leghorn at the onset of egg laying. Our results will expand the list of miRNAs related to the host responses to SE infection in chickens.

\section{Results}

\section{Preliminary analysis of the raw data}

Six libraries were constructed from infected (I) and noninfected $(\mathrm{N})$ chickens, three in each group. The average number of total reads of three libraries obtained from I and $\mathrm{N}$ chickens were 12,476,156 and 10,866,976, respectively, and the filtered clean reads were 5,078,218 and $2,411,757$, respectively (Table 1). In the I group, 3,456,099 clean reads were exactly matched to the chicken genome, 525,400 1-mismatched and 108,131 2-mismatched. In the $\mathrm{N}$ group, 1,722,678 clean reads were exactly matched to chicken reference genome, 149,372 1-mismatched and 47,761 2-mismatched. Altogether, $80.5 \%(4,089,630)$ and $79.6 \%(1,919,811)$ of clean reads were mapped to the genome in the I and $\mathrm{N}$ group, respectively. The miRNAs with $21 \mathrm{nt}$ in length were most abundant followed by $22 \mathrm{nt}$ in both I and $\mathrm{N}$ groups.

Number of reads in each chromosome (Chr) was counted (Additional file 1 and Fig. 1). In general, number of read counts in I group was more than that in $\mathrm{N}$ group in each chromosome. For the I group, there were

Table 1 Number of reads in the infected and non-infected groups

\begin{tabular}{lll}
\hline Item & Infected & Non-infected \\
\hline Total read & $12,476,156$ & $10,866,976$ \\
Clean data & $5,078,218$ & $2,411,757$ \\
Exact matched reads & $3,456,099$ & $1,722,678$ \\
1-mismatch reads & 525,400 & 149,372 \\
2 mismatch reads & 108,131 & 47,761 \\
Total matched reads & $4,089,630$ & $1,919,811$ \\
\hline
\end{tabular}

$769,514,929,207$ and 810,295 clean reads matched on Chr1, Chr3, and Chr13, which account for 19.81, 18.82 and $22.72 \%$ of total clean reads, respectively. For the $\mathrm{N}$ group, there were 367,058 and 693,717 clean reads matched on Chr1 and Chr3, which account for 19.12 and $36.13 \%$ of total clean reads, respectively. The count of matched clean reads on Chr13 in I group was eight folds more than that in $\mathrm{N}$ group.

\section{MiRNA identification and genome distribution}

To identify the miRNAs, small RNA sequences obtained by deep sequencing were aligned with known miRNA sequences in the miRBase and Rfam (http://pfam.xfam.org). The novel miRNAs were predicted through miRDeep. There were 598 miRNAs including 194 novel miRNAs identified. The number of identified miRNAs in each chromosome was counted (Fig. 2). The chromosomes could be divided into three clusters based on the number of mapped miRNAs: (1) Chr18, Chr22, Chr24, Chr25, Chr27, less than ten miRNAs in each chromosome, (2) Chr5, Chr6, Chr8-12, Chr19, Chr21, Chr23, Chr26 and Chr28, 10-20 miRNAs in each chromosome, (3) Chr1-4, Chr7, Chr13-17, Chr20 and ChrZ, more than 20 miRNAs in each chromosome. There were 77 miRNAs mapped on Chr1 and only two miRNAs on Chr22, respectively. The density distribution of miRNAs across chromosomes showed that number of miRNAs in 1Mbp DNA in each chromosome ranged from 0.19 (ChrW) to 2.77 (Chr21) (Additional file 2). The density of miRNAs in Chr1-12 was lower than one miRNA per $1 \mathrm{Mbp}$ DNA. The density of miRNAs in Chr21, Chr23, Chr26 and Chr28 were more than two miRNAs per $1 \mathrm{Mbp}$ DNA. There were $20-60 \%$ of known miRNAs in the miRBase observed in each chromosome except for the Chr21 (91.67\%), Chr23 (73.33\%), Chr18 (15.79\%) and ChrUn (12.5\%).

\section{Differentially expressed miRNAs responding to SE infection}

The differentially expressed miRNAs between I and $\mathrm{N}$ groups were identified through edgeR package. There were 37 miRNAs significantly differentially expressed between I and $\mathrm{N}$ groups including 19 known miRNAs and 18 potentially novel miRNAs $(P<0.05$ with false discovery rate (FDR) of 0.49 and fold change $>2$ ) (Table 2). There were 22 miRNAs up-regulated post SE infection, which included 15 known and seven novel miRNAs. The highest fold change (71.24) was observed for gga-miR-490- $5 \mathrm{p}$ and the lowest fold change (2.06) for gga-miR-193b-3p. Fifteen miRNAs were down-regulated in the comparison of $\mathrm{I} / \mathrm{N}$, which included four known and 11 novel miRNAs. The highest fold change (15.13) was observed for gga-miRchr17_13654 and the lowest (2.01) for gga-miRchr15_12378. Gga-miR-490-5p, gga-miR-chr13_10137 and gga-miR-chrUn_AADN03024004_45551 were unique to 


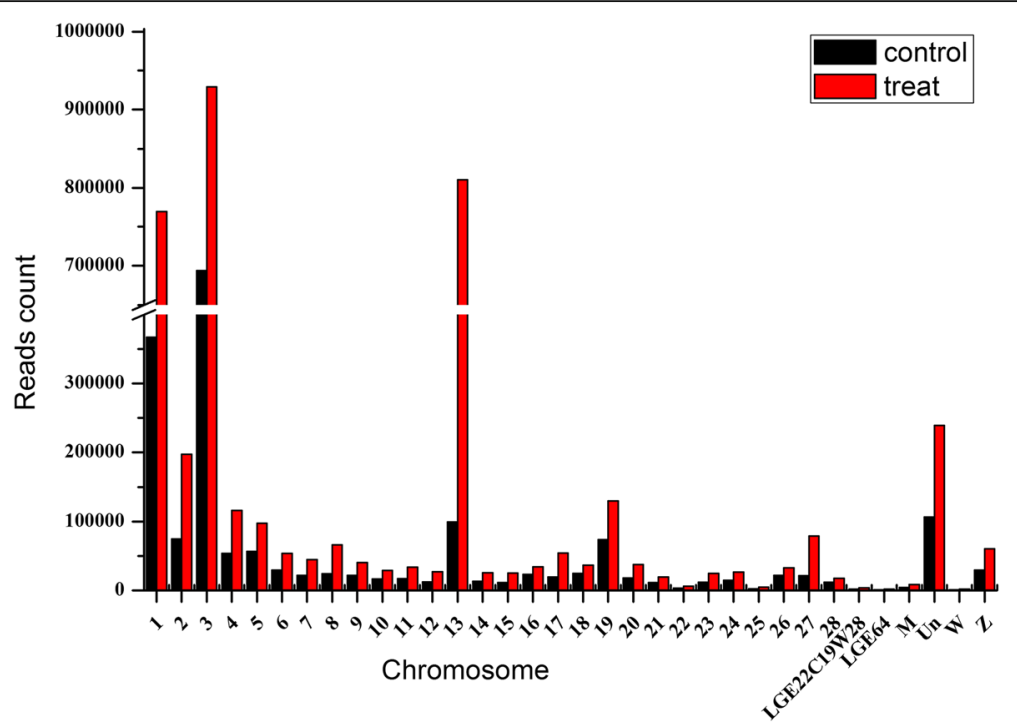

Fig. 1 The distribution of clean reads in the genome

the infected chickens. More than half of significantly differentially expressed miRNAs (17/37) were located on Chr110.

The heat map and hierarchical clustering demonstrated that the miRNA profiles from I and $\mathrm{N}$ groups were distinct (Fig. 3). The infected and non-infected groups were separated distinctly. The differentially expressed miRNAs were clustered into two groups. MiRNAs in group one showed higher expression in the $\mathrm{N}$ group but lower expression in I group, which including gga-miR-34a-5p, gga-miR-215-5p, and gga-miR-1662. MiRNAs in group two showed higher expression in I group but lower expression in $\mathrm{N}$ group including ggamiR-125b-3p, gga-miR-1416-5p and gga-miR-125b-5p.

\section{MiRNA target gene identification}

To investigate the biological role of the differentially expressed miRNAs, the potential target genes were predicted using Miranda algorithm with the Vienna package. In total, 2897 unique target genes regulated by those differentially expressed miRNAs were predicted, in which, 841 genes were uniquely regulated by up-regulated miRNAs (G1), 636 genes were uniquely regulated by downregulated miRNAs (G2), and 1420 were co-regulated by

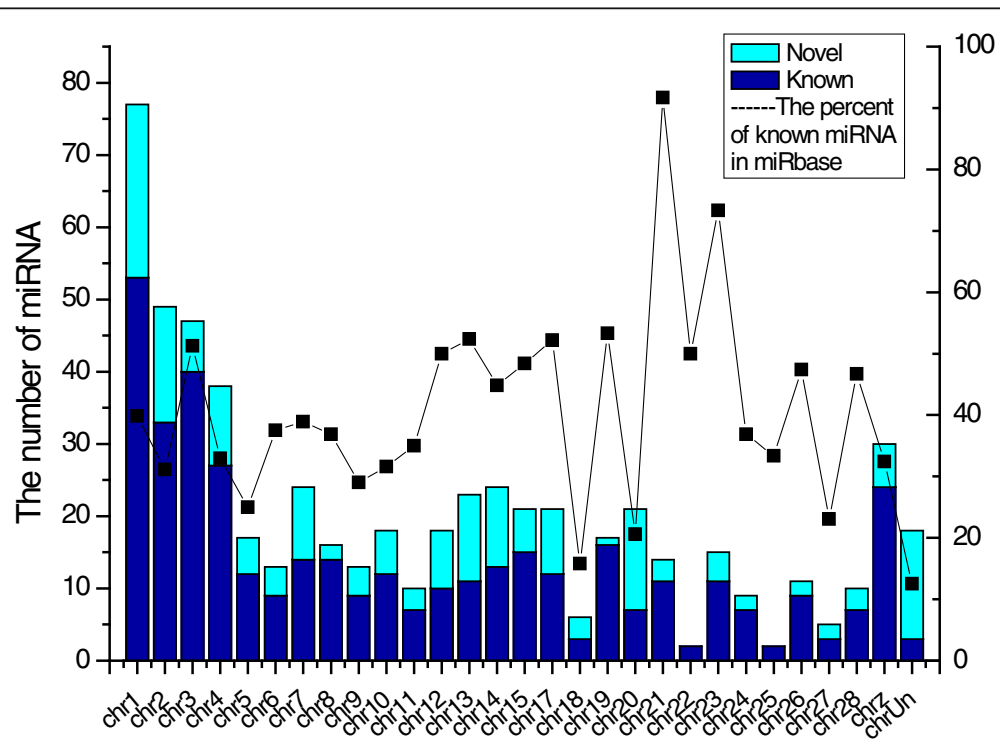

Fig. 2 The distribution of miRNAs in genome 
Table 2 Differentially expressed miRNAs between infected and non-infected chickens $(P<0.05$, Fold change $>2)$

\begin{tabular}{|c|c|c|c|c|}
\hline miRNA & $\begin{array}{l}\text { Read counts in } \\
\text { non-infected }\end{array}$ & $\begin{array}{l}\text { Read counts } \\
\text { in infected }\end{array}$ & Fold change(I/N) & Chromosome position \\
\hline gga-miR-490-5p & 0 & 52.25 & $-{ }^{a}$ & chr1:58018956-58019049 \\
\hline gga-miR-chr13_10137 & 0 & 21.65 & $-{ }^{a}$ & chr13:1853152-1853219 \\
\hline gga-miR chrUn_AADN03024004_45551 & 0 & 14.83 & $-{ }^{\mathrm{a}}$ & chrUn_AADN03024004:12315-12384 \\
\hline gga-miR-216a & 13.32 & 208.79 & 15.63 & chr3:301576-301682 \\
\hline gga-miR-217-5p & 35.76 & 250.64 & 6.65 & chr3:298908-299015 \\
\hline gga-miR-193a-3p & 31.91 & 153.08 & 4.27 & -二-—- \\
\hline gga-miR-1a-3p & 383.04 & 1784.26 & 4.26 & chr20:8472264-8472335 \\
\hline gga-miR-1b-3p & 298.63 & 1358.11 & 4.12 & chr23:4294311-4294375 \\
\hline gga-miR-chr6_35375 & 71.63 & 300.31 & 4.05 & chr6:2271072-2271138 \\
\hline gga-miR-490-3p & 283.61 & 1123.92 & 3.76 & chr1:58018956-58019049 \\
\hline gga-miR-133a-3p & 11115.44 & 43083.62 & 3.51 & chr2:102176852-102176939 \\
\hline gga-miR-133c-3p & 9130.66 & 33983.52 & 3.38 & chr23:4294450-4294529 \\
\hline gga-miR-133b & 8841.86 & 32748.91 & 3.36 & chr3:107209162-107209246 \\
\hline gga-miR-9-3p & 27.29 & 94.26 & 3.24 & chr28:3378846-3378934 \\
\hline gga-miR-chr13_10219 & 5157.10 & 16050.59 & 2.93 & chr13:7833540-7833603 \\
\hline gga-miR-chr13_10222 & 907675.18 & 2759302.93 & 2.86 & chr13:7834142-7834200 \\
\hline gga-miR-chr15_12339 & 221046.01 & 604996.23 & 2.59 & chr15:463481-463561 \\
\hline gga-miR-chr3_27485 & 334.10 & 844.81 & 2.41 & chr3:65480575-65480649 \\
\hline gga-miR-1416-5p & 177.32 & 420.27 & 2.30 & chrZ:34781500-34781589 \\
\hline gga-miR-125b-3p & 1026.62 & 2508.62 & 2.29 & chr1:98380667-98380757 \\
\hline gga-miR-125b-5p & 6220.19 & 14048.45 & 2.10 & chr1:98380667-98380757 \\
\hline gga-miR-193b-3p & 216.46 & 492.77 & 2.06 & chr14:762080-762163 \\
\hline gga-miR-chr15_12378 & 10551.58 & 5882.44 & -2.01 & chr15:4870074-4870145 \\
\hline gga-miR-215-5p & 6638307.42 & 3618379.46 & -2.02 & chr3:18143688-18143793 \\
\hline gga-miR-147 & 3180.31 & 1712.26 & -2.10 & chr10:10170160-10170230 \\
\hline gga-miR-1662 & 2111.61 & 1000.82 & -2.39 & chr2:1818924-1818997 \\
\hline gga-miR-chr7_36925 & 1908.29 & 858.02 & -2.50 & chr7:6845145-6845212 \\
\hline gga-miR-chr2_16700 & 141.08 & 49.79 & -2.78 & chr2:73136006-73136063 \\
\hline gga-miR-34a-5p & 278.17 & 99.89 & -2.93 & chr21:3266631-3266740 \\
\hline gga-miR-chr10_6644 & 27.94 & 3.81 & -3.85 & chr10:7087285-7087353 \\
\hline gga-miR-chr1_5477 & 36.18 & 4.44 & -4.69 & chr1:152779416-152779543 \\
\hline gga-miR-chr10_6507 & 51.26 & 13.08 & -4.88 & chr10:1689882-1689969 \\
\hline gga-miR-chrUn_AADN03024004_45550 & 16.72 & 1.27 & -5.56 & chrUn_AADN03024004:1006-1069 \\
\hline gga-miR-chr20_19772 & 62.48 & 14.47 & -6.10 & chr20:10132999-10133045 \\
\hline gga-miR-chr20_19953 & 281.37 & 24.37 & -15.02 & chr20:1049342-1049435 \\
\hline gga-miR-chr20_19955 & 281.37 & 24.37 & -15.02 & chr20:1153806-1153899 \\
\hline gga-miR-chr17_13654 & 40.24 & 1.27 & -15.13 & chr17:6752602-6752677 \\
\hline
\end{tabular}

${ }^{a}$ Specifically expressed in infected group

both up and down-regulated miRNAs (G3). One hundred and seventy-six immune-related genes were retrieved from the Ensembl BioMart database [20]. In total, 587 pairs of interaction between miRNAs and immune-related genes were obtained (Additional file 3). Gga-miR-34a-5p interacted with the greatest number of immune-related genes
(46), while the gga-miR-215-5p only interacted with one immune-related gene.

\section{Gene ontology (GO) analysis for target genes}

To demonstrate the function of target genes of differentially expressed miRNAs, functional annotation was 


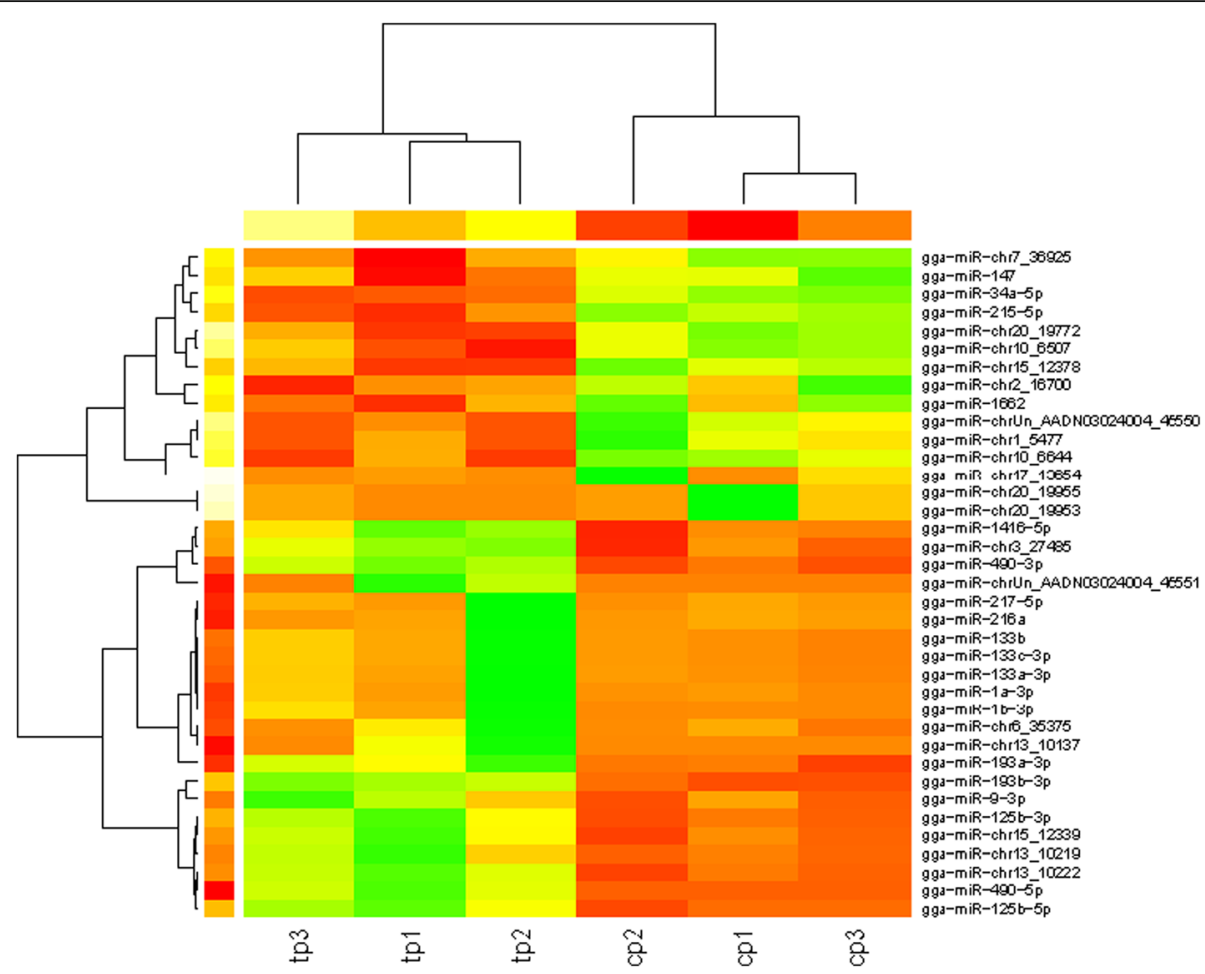

Fig. 3 The heatmap of the differentially expressed miRNAs. Note: The heat map was computed using a function of heatmap.2 in gplots by $\mathrm{R}$ platform. The green indicated higher miRNA expression level and the red showed lower miRNA expression level. Tp1, tp2 and tp3 were the sample pools in the I group, $\mathrm{cp} 1, \mathrm{cp} 2$ and $\mathrm{cp} 2$ were sample pools in the $\mathrm{N}$ group

performed through DAVID (The Database for Annotation, Visualization and Integrated Discovery) for the target genes in the G1, G2 and G3 groups, respectively (Additional file 4). There were 118, 73 and 178 GO BP (biological process) terms significantly enriched in G1, G2 and G3, respectively $(P<0.05)$. The GO BP terms were categorized by CateGOrizer using "Immune System Gene Classes" GO classification (Fig. 4). For target genes in G1, the enriched GO BP terms were categorized into ten ancestral classes, which could be divided into three groups: 1) immune-related function including death, apoptosis, cell adhesion, stress response, response to abiotic stimulus, regulation of apoptosis, and response to external stimulus occupied $61.54 \%$ of all ancestral classes, 2) metabolism-related including carbohydrate metabolism, protein metabolism, and catabolism, occupied $30.76 \%$ of all ancestral classes, 3) cell adhesion occupied $7.69 \%$ of all ancestral classes. For target genes in G2, the enriched GO BP terms were categorized into 12 ancestral classes. The immune-related classes including stress response, death, response to abiotic stimulus, lymphocyte activation, apoptosis, $\mathrm{T}$ cell activation, lymphocyte differentiation, regulation of apoptosis, occupied 36.83\% of all ancestral classes. The metabolism-related classes including catabolism, protein metabolism, carbohydrate metabolism, occupied $60.53 \%$ of all ancestral classes. For target genes in G3, the enriched BP terms were categorized into 20 ancestral classes, the immune-related classes occupied $80.58 \%$ of all ancestral classes, and metabolism-related classes occupied $15.28 \%$.

\section{KEGG Pathway analysis}

For the target genes in the G1, only one pathway of endocytosis was significantly enriched $(P<0.05)$ with the fold enrichment of 1.65, which included 18 target genes. For target genes in the G2, five pathways were significantly enriched, which were ECM-receptor interaction, glycolysis/gluconeogenesis, focal adhesion, melanogenesis and proteasome. Proteasome had the highest fold enrichment of 3.53 and focal adhesion included the largest number of target genes (16). For target genes in the G3, Cell cycle and SNARE interactions in vesicular transport were significantly enriched and associated with 27 and 9 genes, respectively (Table 3).

\section{Protein-protein Interacting Network of the immune- related target genes}

The Reactome FIViz (http://f1000research.com/articles/ 3-146/v2) app in Cytoscape was used to examine the potential protein-protein interactions of the 176 immunerelated genes encoded proteins [21]. Through Cytoscape, there were 98 proteins accepted by Reactome FIViz and 

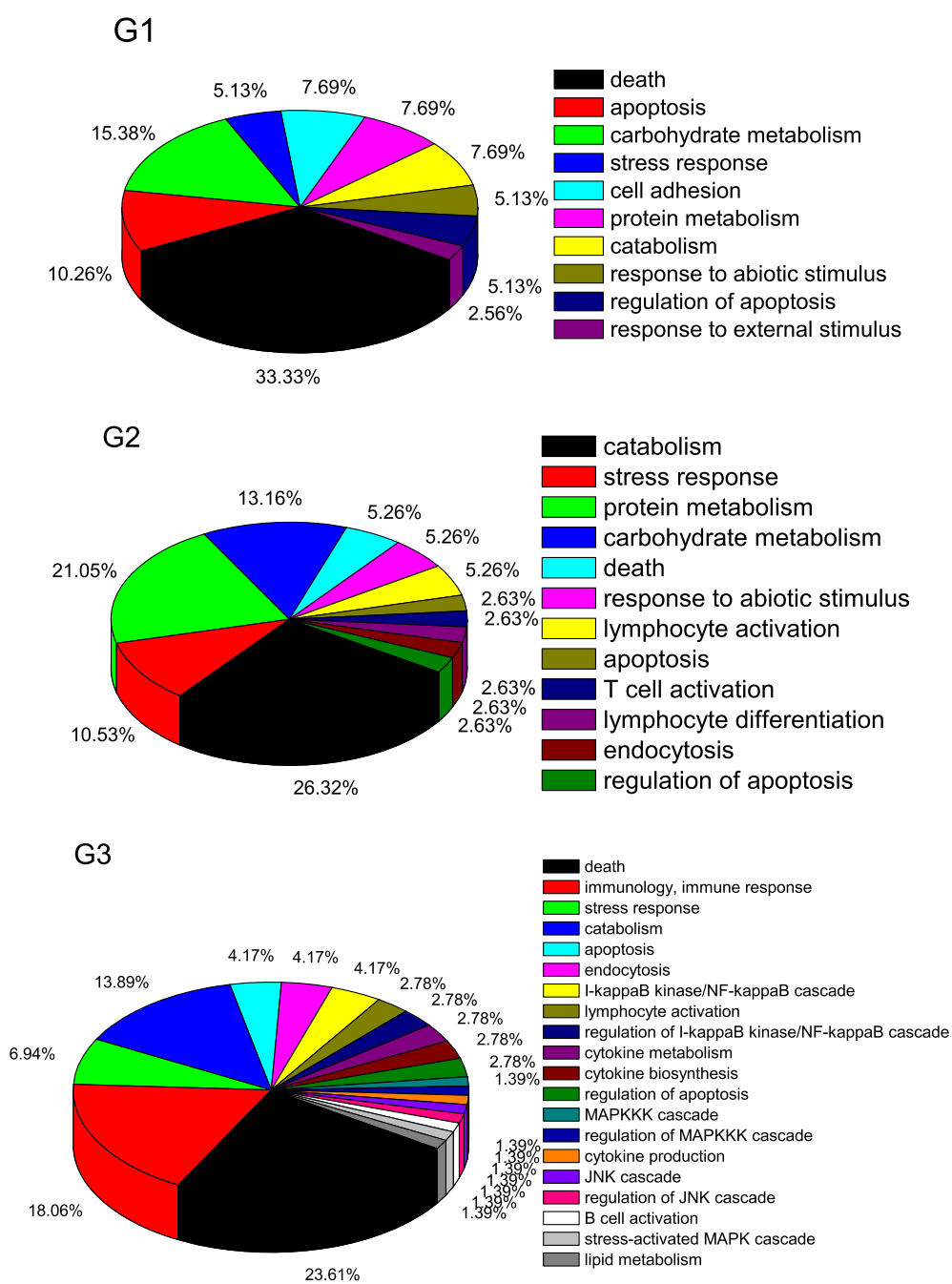

Fig. 4 The classification of GO BP terms associated with target genes. Note: G1, genes uniquely regulated by up-regulated miRNAs; G2, genes uniquely regulated by down-regulated miRNAs; G3, genes co-regulated by both up and down-regulated miRNAs

clustered into six network modules (Fig. 5). The top ten proteins interacted with more than 15 other proteins were illustrated in the center of the network. JUN protein interacted with 32 proteins, and LYN and JAK with 21 proteins, respectively.

\section{Validation of differentially expressed miRNAs}

The relative expression level of 12 differentially expressed miRNAs regulating immune-related genes was validated through quantitative real-time PCR (qRT-PCR). The specific primers for those 12 miRNAs (seven up-regulated miRNAs (including two novel miRNAs) and five downregulated miRNAs (including two novel miRNAs)) were listed in Additional file 5. The results showed that all the miRNAs selected for qRT-PCR validation were significantly differentially expressed. All the results in qRT-PCR were consistent with those obtained from the sequencing except for gga-miR-193b-3p which had reverse regulatory direction (Table 4). The gga-miR-1416-5p and gga-miR125b-5p were up-regulated. The gga-miR-1662 and ggamiR-34a-5p were down-regulated after SE infection.

\section{Target genes expression}

Twelve immune-related target genes of 4 differentially expressed miRNAs (gga-miR-1416-5p, gga-miR-1662, ggamiR-125b-5p and gga-miR-34a-5p) were selected to detect the relative expression using qRT-PCR. The potential regulation was listed in Additional file 6. The specific primers were listed in Additional file 7 . The results showed that eight target genes were significantly differentially expressed between I and N groups (Fig. 6). NOTCH2, THBS1, RIPK2, $I G J$ and TLR1LA were significantly up-regulated $(P<0.05)$ and CCL4, TLR21 and BCL10 were significantly downregulated following SE infection. Five of eight target genes had reversely regulatory direction with their regulating miRNAs following SE infection. 
Table 3 The significantly enriched pathways in three groups of target genes $(P<0.05)$

\begin{tabular}{|c|c|c|c|c|c|}
\hline & Pathway & No. of genes & $P$ value & Fold enrichment & Genes \\
\hline$\overline{\mathrm{G} 1}$ & Endocytosis & 18 & 0.040 & 1.65 & $\begin{array}{l}\text { CHMP2A, HRAS, CLTC, CHMP2B, EPS15, SMAP2, AP2A2, TFRC, } \\
\text { RABEP1, CXCR4, WWP1, NTRK1, MDM2, IL2RG, PDCD6IP, EGF, } \\
\text { EHD3, AP2M1 }\end{array}$ \\
\hline \multirow[t]{5}{*}{ G2 } & ECM-receptor interaction & 10 & 0.007 & 2.86 & $\begin{array}{l}\text { CD47, COL4A2, ITGA8, ITGAV, COL6A3, ITGA3, VTN, SDC2, } \\
\text { SDC3, SPP1 }\end{array}$ \\
\hline & Glycolysis/Gluconeogenesis & 7 & 0.017 & 3.32 & LDHB, GPI, TPI1, LDHA, PGM1, DLD, PGAM1 \\
\hline & Focal adhesion & 16 & 0.018 & 1.91 & $\begin{array}{l}\text { COL4A2, MAP2K1, ACTN1, ITGA3, VTN, PPP1CC, CDC42, } \\
\text { CCND1, ITGA8, ITGAV, COL6A3, ILK, RHOA, MAPK9, FIGF, SPP1 }\end{array}$ \\
\hline & Melanogenesis & 10 & 0.020 & 2.43 & $\begin{array}{l}\text { WNT5A, KRAS, MAP2K1, CALM, MAP2K2, ADCY5, FZD1, } \\
\text { CAMK2D, WNT6, FZD7 }\end{array}$ \\
\hline & Proteasome & 6 & 0.024 & 3.53 & PSMC3, PSMA4, PSMC2, PSMD3, PSME3, PSMD4 \\
\hline \multirow[t]{2}{*}{ G3 } & Cell cycle & 27 & $3.68 \mathrm{E}-04$ & 2.04 & $\begin{array}{l}\text { E2F1, YWHAZ, E2F4, E2F5, ANAPC10, CIP1, CDKN2B, TFDP2, } \\
\text { BUB1, CCNA2, RBL2, YWHAB, SKP2, RB1, SKP1, CDC27, MCM5, } \\
\text { ATM, CDC25A, WEE1, CDK3, YWHAG, YWHAH, CCND3, HDAC1, } \\
\text { PLK1, YWHAQ }\end{array}$ \\
\hline & $\begin{array}{l}\text { SNARE interactions in vesicular } \\
\text { transport }\end{array}$ & 9 & 0.041 & 2.21 & $\begin{array}{l}\text { STX6, STX17, USE1, BET1L, SEC22B, VAMP3, GOSR2, SNAP23, } \\
\text { GOSR1 }\end{array}$ \\
\hline
\end{tabular}

Note: G1 means the unique target genes of up-regulated miRNAs, G2 means the unique target genes of down-regulated miRNAs, G3 means the target genes regulated by both up and down-regulated miRNAs

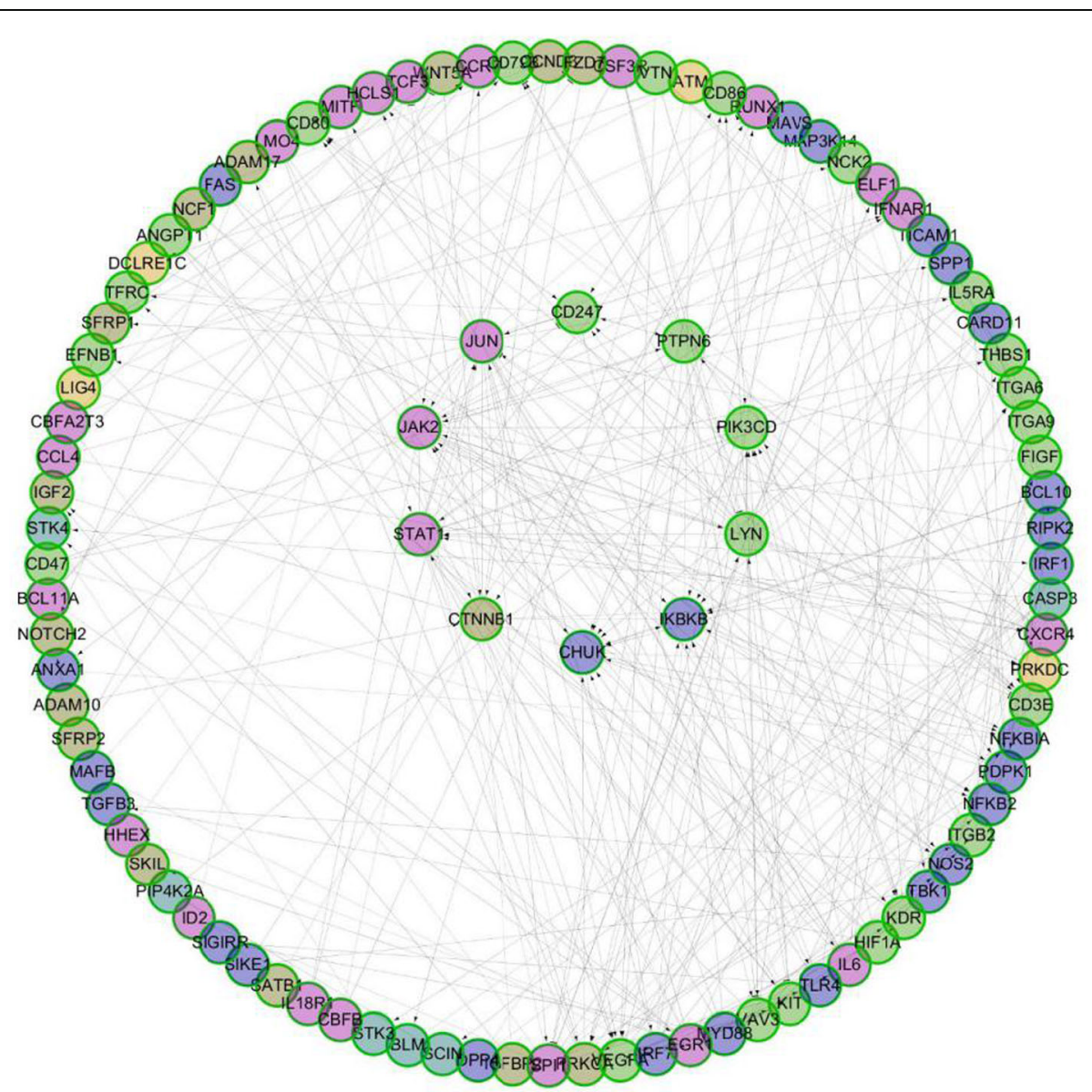

Fig. 5 Protein-protein interaction network of the immune-related target genes 
Table 4 Fold change of miRNAs obtained from qRT-PCR and Solexa Sequencing

\begin{tabular}{llc}
\hline miRNA & qRT-PCR & Sequencing \\
\hline gga-miR-34a-5p & $0.363^{*}$ & $0.342^{*}$ \\
gga-miR-chr15_12378 & $0.382^{* *}$ & $0.498^{*}$ \\
gga-miR-chr7_36925 & $0.274^{* *}$ & $0.400^{*}$ \\
gga-miR-1662 & $0.418^{* *}$ & $0.419^{*}$ \\
gga-miR-215-5p & $0.416^{* *}$ & $0.495^{*}$ \\
gga-miR-133b & $2.283^{* *}$ & $3.359^{*}$ \\
gga-miR-chr13_10219 & $2.229^{* *}$ & $2.931^{* *}$ \\
gga-miR-chr13_10222 & $1.810^{*}$ & $2.862^{* *}$ \\
gga-miR-1b-3p & $2.808^{* *}$ & $4.115^{* *}$ \\
gga-miR-1416-5p & $2.602^{* *}$ & $2.297^{*}$ \\
gga-miR-193b-3p & $0.575^{*}$ & $2.060^{*}$ \\
gga-miR-125b-5p & $2.335^{* *}$ & $2.095^{*}$ \\
\hline
\end{tabular}

Note: ${ }^{*}, P<0.05 ;{ }^{*}, P<0.01$

\section{Discussion}

MiRNAs play a vital role in regulating immunity induced by bacteria [22-24]. Regulation of miRNA expression responding to bacterial infection is emerging as a crucial part of the host response to infection [22]. The onset of egg laying is a complex physiological process when physiological and hormonal stress occurs. Previously, we have studied the splenic gene expression in laying hens through microarray [25]. In the current study, cecal miRNA profiling in the response to SE infection in laying hens has been identified through Solexa sequencing. In total, 404 known miRNAs and 194 potential novel miRNAs were expressed in six libraries.
MiRNA distribution varies widely in different chromosome in different species, and chromosomes with higher number of miRNAs most likely play regulatory roles in several cellular processes including disease. MiRNAs associated with cancer and cardiovascular disease are mostly located in Chr1, 14, 19, and X in human [26]. There is a significant association between the chromosomal location of miRNAs and those of cancer susceptibility loci in mouse [27]. There are $39.66 \%$ miRNA genes located on chicken chromosome $1,2,3$, and 4 [26]. The skew distribution of miRNAs has been observed following SE infection in the current study. Majority of miRNAs responding to SE infection located on Chr1, 2, 3, 10, 13, and 20. There was no miRNAs observed in chicken Chr16 which is consistent with the current results from miRBase database (http://www.mirbase.org).

SE infection stimulates the miRNAs expression. Thirtyseven miRNAs were differentially expressed following SE infection. Read counts in the infected group were higher than those in the non-infected group (Fig. 1). In the current study, more up-regulated miRNAs were observed at 7 days post infection (dpi) (22 up-regulated vs. 15 downregulated). More miRNAs are up-regulated at 2 days post infection with S. Typhimurium in piglet which is consistent with current miRNA profiling [28]. The similar response to Gram-negative bacterial infection could be regulated by miRNAs. MiRNA regulation in immune response is influenced by genetic background. It has been reported that opposite miRNA profiling was found between SPF layer and broiler chickens infected with AIV (Avian Influenza Virus) $[19,29]$. Functionally, the miRNAs target mRNAs and trigger either translation repression or RNA degradation in animal [30]. It has been reported that the complicated interaction between immune system and metabolism exists

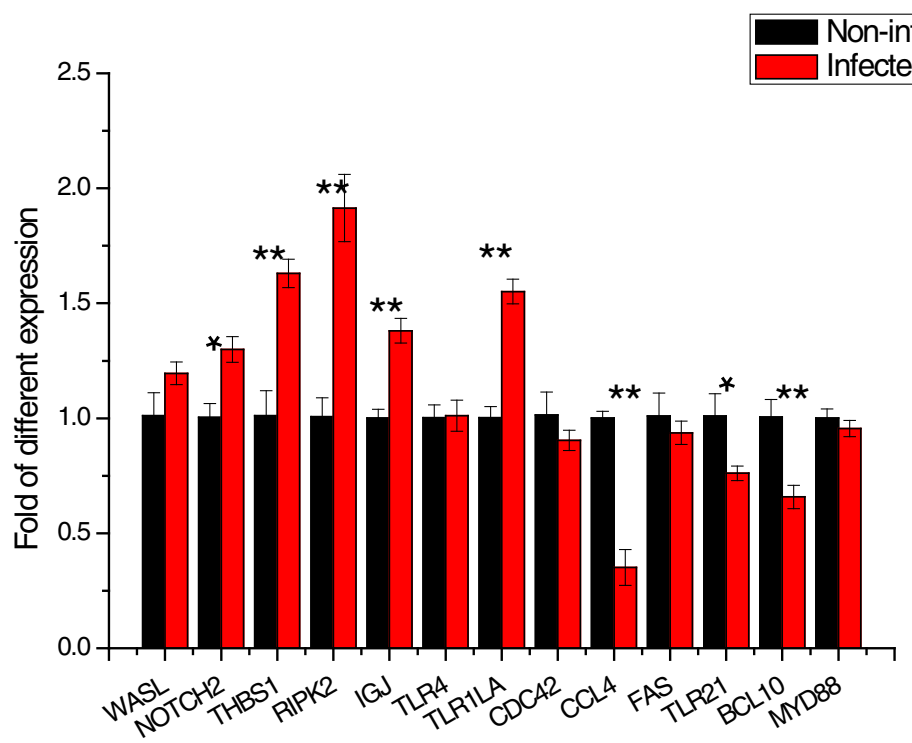

Fig. 6 Qualification of immune-related target genes of differentially expressed miRNAs 
during SE infection in laying hens [31]. Maintaining metabolic homeostasis requires a balanced immune response. The perturbation of this equilibrium could result in pathological situations [32]. It has been reported that miRNAs are fine-tuners of metabolic processes [33] and could generate a bidirectional functional link between metabolism and pathogenesis [32]. In the current study, target genes of upregulated miRNAs were associated with stronger immune function than metabolism function. Target genes of downregulated miRNAs were associated with stronger metabolism compared with immune function. The target genes regulated by both up- and down-regulated miRNAs were also associated with stronger immune function than metabolism (Fig. 4). The target genes of up- and down regulated miRNAs may buffer the homeostasis between immune response and metabolism. Those miRNAs may contribute to buffer the gene expression of target genes $[16,32]$. The immune function was repressed at the onset of egg laying $[25,34,35]$. The results herein suggested that miRNAs could regulate the interaction between immune response and metabolism on day 7 post SE infection in laying hens.

It is noteworthy that the Proteasome pathway had the highest fold enrichment in the enriched pathways. The proteasome is a protein-destroying apparatus involved in many essential cellular functions including antigen processing for appropriate immune responses and inflammatory responses [36-38]. The nonclassical class Ib molecule Qa-1b is a dominant restricting element to the recognition of CD8+ T Cells which is proteasome-dependent during Salmonella infection [39]. Type III protein secretion systems (T3SS) effector protein activities of Salmonella are temporally regulated by proteasome-dependent protein degradation [40]. The LMP2 protein, a subunit of the cytosolic proteasome complex, is up-regulated after invasion of HLA-B27transfected HeLa cells by Salmonella typhimurium [41]. Both Salmonella enteritidis and Salmonella typhimurium are gram-negative bacteria. This could suggest that proteasome is an important defense system to protect against bacterial colonization regulated by miRNAs in chicken.

MiR-34a-5p was down-regulated and potentially targeted 46 immune-related genes in chicken after SE infection. MiR-34a was associated with the cellular senescence, it was strongly up-regulated and targeted the important proto-oncogene $M Y C$ during B-RAFinduced senescence [42]. MiR-34a may play an important role in the loss of oxidative defense in rat liver during aging through suppressing the expression of Sirt1, Mgst1, Sp1 and Nrf2 [43]. Bcl-2 and the Foxp1 transcription factor, which were required for early $B$ cell development [44], are the direct target of miR-34a, so it was hypothesized that miR-34a plays an important role in B cell development [45-47]. MiR-34a causes a partial block in B cell development, whereas its knockdown results in increased B cells development in rat bone marrow [48]. CCL4, IL8L1 and CDC42 were contained in the Salmonella infection pathway and were all targeted by miR-34a. CCL4 gene is significantly upregulated at different time points $(1,2,4$, and $8 \mathrm{~h})$ after SE endotoxin treatment [49]. CDC42 could activate the Arp $2 / 3$ by N-WASP (Neural Wiskott-Aldrich syndrome protein) to mediate actin polymerization in the invasion of SE [50-53]. It has been reported that mir-34a exclusively expressed in AIV infected chicken lung with targeting 14 immune-related genes and four AIV genes [29]. Although there were no SE genes regulated by mir-34a predicted, the down-regulation of miR-34a may conduce to B cell development in the response to SE infection in chickens.

Gga-miR-1416 is located in the intron of MAMDC 2 (MAM domain containing 2) on ChrZ. Gga-miR-1416 is up-regulated in both layer and broiler following AIV infection suggests that it responds to AIV infection across diverse genetic lines [29]. Six immune-related genes were potentially targeted by gga-miR-1416-5p including BCL10, NFKBIA and TLR21, which were important in the response to bacterial infection. $B C L 10$ mediates the LPS-induced activation of $N F-\kappa B$ and IL8 in normal human intestinal epithelial cells [54]. Chicken TLR21 is involved in the recognition of bacterial components and Salmonella in the innate immune response [55]. The expression of TLR 21 was decreased in the duodenum, jejunum, ileum, ceca and large intestine of broilers following SE infection [56]. In the current study, the expression of TLR 21 was significantly down-regulated and had reversely regulatory direction with the regulating gga-miR-1416-5p post SE infection. Gga-miR-1416-5p could play an important role in response to SE infection through regulating the target genes.

The interaction of miRNA with target genes plays an important role in many biological functions. Toll-like receptors $(T L R)$ recognizes specific patterns of microbial components and participate in the innate immunity and antigen-specific adaptive immunity [57]. As one of the main pattern recognition receptors, TLR can identify the pathogen to activate the immune cells response and induce to produce type I interferon and a series of proinflammatory cytokines [58]. TLR1LA could combine with $T L R 2$ to form a dimer and efficiently identify bacterial peptidoglycan and lipoprotein [59]. TLR1LA is significantly up-regulated at the ileum, cecum and colon at $24 \mathrm{~h}$ post SE infection in day-old chickens [56]. GgamiR-1662 was significantly up-regulated in chicken lung following AIV infection [29]. Gga-miR-1662 was downregulated while its target gene TLR1LA was up-regulated in the response to SE infection in the current study. The interaction of gga-miR-1662 with TLR1LA could be important in the respond to SE infection in chicken. Further study is warranted to verify the function of interaction between gga-miR-1662 and TLR1LA. 


\section{Conclusions}

MiRNAs mediate the homeostasis between metabolism and immunity in the response to SE infection at the onset of egg laying. The gga-miR-34a-5p, gga-miR-1416-5p and gga-miR-1662 could play important roles in SE infection through regulating their target genes. The finding herein will pave the foundation for the studies of microRNA regulation in SE infection in laying hens.

\section{Methods}

\section{Animals and SE challenges}

One hundred 20-week old SE negative White Leghorn layers were used in the current study. Chickens were randomly divided into two groups with equal number, infected group (I) and non-infected $(\mathrm{N})$ group. At the first day, chickens in the infected group were fed with $30 \mathrm{~g}$ feed containing $5.8 \times 10^{8} \mathrm{cfu} \mathrm{SE}$ at a time and the non-infected birds were fed with regular feed as described previously (Wu et al., [25]). Chickens in each group were sacrificed by cervical dislocation at 7 days post infection (dpi). The cecum samples were aseptically harvested from each chicken and put into RNAlater solution (Life technologies, Grand Island, USA) and stored at $-20{ }^{\circ} \mathrm{C}$. All animal procedures were approved by Shandong Agricultural University Animal Care and Use Committee.

\section{Small RNAs library construction and deep sequencing}

In total, nine infected and six non-infected birds were randomly selected for further RNA isolation. Total RNA was isolated from each individual sample using Trizol following the manufacture's protocol (Life technologies, Grand Island, USA). The RNA integrity and concentration were checked and measured using gel electrophoresis and Nanodrop (Thermo Fisher Scientific, Wilmington, USA).

Six small RNA libraries were pooled from I and $\mathrm{N}$ groups, three in each group. Each small RNA library from I (tp1, tp2, tp3) and N (cp1, cp2, cp3) group consisted of three and two individual samples with equal amount of RNA, respectively. The small RNA pools were purified and enriched using denaturing polyacrylamide gel electrophoresis. A pair of Illumina proprietary adaptors was ligated to their 5' and 3' ends, followed by reverse transcription and cluster generation using TruSeq Small RNA Library Preparation Kit (Illumina Inc., San Diego, USA). Subsequently, the libraries were sequenced by Illumina Hiseq 2500 according to the manufacturer's instructions at Genergy Inc (Shanghai, China).

\section{Basic data processing}

The small RNA sequence reads were pre-processed using FASTX-Toolkit to filter low-quality reads and trim adaptor. After filtering adaptor sequences and removing contaminated reads, the clean reads were matched to chicken reference genome using the Bowtie [60]. The first 15 bp of the read was exactly matched. Two mismatched bases were accepted on the rest nucleotides of the read. The filtered sequences were matched with miRBase (http://mirbase.org/) to search for known miRNAs with exact matches. The unmatched data sets were aligned with chicken genomic sequence (Gallus_gallus4.0) and predict the novel miRNAs using miRDeep [61].

\section{Differential expression analysis of miRNAs}

To compare miRNAs expression level between I and $\mathrm{N}$ groups, read count of each identified miRNA was normalized to the total number of reads in each given sample. The differentially expressed miRNAs were identified through edgeR package with False discovery rate multiple testing correction [62]. The $P<0.05$ and fold change $>2$ was considered as significant difference.

\section{Target prediction and Gene Ontology (GO) enrichment analysis}

The target genes of those significantly differentially expressed miRNAs were predicted using Miranda algorithm through the Vienna package [63, 64]. Functional annotation of GO and pathway analysis for those target genes of miRNAs were performed through DAVID 6.7 [65-67]. Significant over-representation is based on a Fisher Exact statistical methodology similar to that described by Al-Shahrour et al [68]. CateGOrizer was used to categorize the significantly enriched GO BP (biological process) terms [69].

\section{Protein-protein Interaction Network Analysis of the immune-related target genes}

We converted the gene IDs of 176 immune-related target genes to the gene symbols of the encoded proteins using bioDBnet software (www.biodbnet.abcc.ncifcrf.gov/db/db2 db.php\#biodb). Then the gene symbols were mapped onto the chicken functional interaction network found in the Reactome database using the Reactome FI network plug-in in the Cytoscape software [70].

\section{Quantitative real-time PCR of miRNAs and target genes}

The same individual RNA samples used for sequencing were used for quantitative real-time PCR (qRT-PCR). In total, nine infected and six non-infected samples were used. Twelve differentially expressed miRNAs were validated and characterized using qRT-PCR. In brief, $1 \mu \mathrm{g}$ of total RNA was reverse transcribed using Step PrimeScript $^{\oplus}$ miRNA cDNA Synthesis Kit (Perfect Real Time) and amplified using Stratagene MX3000 real-time PCR System with miRNA specific primers (Additional file 5). The qRT-PCR was performed with SYBR green PCR master mix. Small nucleolar RNA U6 was used as endogenous control to normalize RNA input. The $20 \mu \mathrm{L}$ PCR reactions included $10 \mu \mathrm{L}$ SYBR $^{\oplus}$ Premix Ex TaqTM 
II $(2 \times), 0.4 \mu \mathrm{L}$ ROX Reference Dye $(50 \times), 0.8 \mu \mathrm{L}$ UnimiR qPCR Primer $(10 \mu \mathrm{M}), 0.8 \mu \mathrm{L}$ forward qPCR Primer, $2 \mu \mathrm{L}$ cDNA and $6 \mu \mathrm{L} \mathrm{ddH}_{2} \mathrm{O}$. The qPCR amplification conditions were: 1 cycle of $95{ }^{\circ} \mathrm{C}$ for 30s, 40 cycles of $95{ }^{\circ} \mathrm{C}$ for $5 \mathrm{~s}$ and $60{ }^{\circ} \mathrm{C}$ for 30s. All qRT-PCR reactions were performed in triplicate. The relative expression were calculated using $2^{-\triangle \Delta C T}$ method. The student's T-test was performed to examine the significance of miRNA expression between I and $\mathrm{N}$ groups.

Relative expression level of 12 immune-related target genes were quantified using qRT-PCR. The RNA was reverse transcribed to cDNA using TaKaRa Primer Script ${ }^{\mathrm{Tu}}$ RT reagent kit (Perfect Real Time) (TaKaRa, Dalian, China) according to the manufacturer's manual. The qRT-PCR was performed by Stratagene MX3000 real-time PCR System with SYBR green method. The specific primers were designed by primer premier 5.0 according to gene sequence (Additional file 7). The $20 \mu \mathrm{L}$ PCR reactions included $10 \mu \mathrm{l}$ SYBR Primer Ex Taq ${ }^{\mathrm{TM}}(2 \times), 0.4 \mu \mathrm{l}$ forward primer $(10 \mu \mathrm{M}), 0.4 \mu \mathrm{l}$ reverse primer $(10 \mu \mathrm{M}), 0.4 \mu \mathrm{L}$ ROX Reference Dye II (50x), $2 \mu \mathrm{L}$ cDNA, $6.8 \mu \mathrm{L}$ $\mathrm{ddH}_{2} \mathrm{O}$. The conditions of qRT-PCR amplification and data analysis were the same as those used in miRNA qRT-PCR. The chicken $\beta$-actin was used as the internal control.

\section{Additional files}

Additional file 1: The distribution of clean reads in the genome. (DOCX $13 \mathrm{~kb}$ )

Additional file 2: Density distribution of miRNAs across chromosomes. Note: Densities are shown as number of miRNAs per megabase of DNA. (TIFF 256 kb)

Additional file 3: Immune-related target genes and interaction with miRNAs. (XLS $128 \mathrm{~kb}$ )

Additional file 4: Enriched GO BP terms in each group. (XLS $100 \mathrm{~kb}$ ) Additional file 5: Primer information for miRNAs validated by qRT-PCR. (DOCX $17 \mathrm{~kb}$ )

Additional file 6: The potential regulation between miRNAs and target genes. (DOCX $15 \mathrm{~kb}$ )

Additional file 7: The designed primers for genes validated by $\mathrm{QRT}$-PCR. (DOC $43 \mathrm{~kb}$ )

\footnotetext{
Abbreviations

SE: Salmonella enterica serovar Enteritidis; miRNA: MicroRNA; GO: Gene ontology; BP: Biological process; G1: Genes were uniquely regulated by upregulated miRNAs; G1: Genes were uniquely regulated by down-regulated miRNAs; G3: Genes co-regulated by both up and down- regulated miRNAs; NGS: Next generation sequencing; I: Infected; N: Non-infected;

Chr: Chromosome; ChrUn contains clone contigs that can't be confidently placed on a specific chromosome; DAVID: The Database for Annotation, Visualization and Integrated Discovery; KEGG: Kyoto Encyclopedia of Genes and Genomes; qRT-PCR: Quantitative real-time PCR; AIV: Avian influenza virus; TTSS: Type III protein secretion systems; TLR: Toll like receptor; IL: Interleukin; FDR: False discovery rate
}

\section{Funding}

This project was supported by the National High Technology Research and Development Program of China (863 Program) (2011AA100305), the National Natural Science Foundation of China (31172204), Shandong Modern

Agricultural Industry \& Technology System (SDAIT-11-02), Shandong Province Agricultural Seed Project, and China Scholarship Council (201508370050).

\section{Availability of data and materials}

The expression data has been deposited into the Gene Expression Omnibus with the accession number GSE59748.

\section{Authors' contributions}

GW analyzed data, qPCR validation and drafted the manuscript. YQ carried out animal inoculation and sample collection. $\mathrm{XL}$ assisted in data analysis and PCR validation. NY participated in the experiment design. GX assisted in animal experiment design and sample collection. LL participated in the development of the experimental design and assisted in data analysis. $\mathrm{XL}$ provided the concepts of the study, and revised the manuscript. All authors read, edited and approved the final manuscript.

\section{Competing interests}

The authors declare that they have no competing interests.

\section{Consent for publication}

Not applicable.

\section{Ethics approval}

All animal procedures were approved by Shandong Agricultural University Animal Care and Use Committee.

\section{Author details}

${ }^{1}$ College of Animal Science and Technology, Shandong Agricultural University, Tai'an, Shandong 271018, China. ${ }^{2}$ College of Life Science, Shandong Agricultural University, Tai'an, Shandong 271018, China. ${ }^{3}$ College of Animal Science and Technology, China Agricultural University, Beijing 100193, China. ${ }^{4}$ Shandong Provincial Key Laboratory of Animal Biotechnology and Disease Control and Prevention, Tai'an, Shandong 271018, China.

Received: 16 June 2016 Accepted: 12 December 2016

Published online: 13 January 2017

\section{References}

1. Scallan E, Hoekstra RM, Angulo FJ, Tauxe RV, Widdowson MA, Roy SL, Jones $J$, Griffin PM. Foodborne illness acquired in the United States-major pathogens. Emerg Infect Dis. 2011;17(1):7-15.

2. Scallan E, Hoekstra RM, Mahon BE, Jones TF, Griffin PM. An assessment of the human health impact of seven leading foodborne pathogens in the United States using disability adjusted life years. Epidemiol Infect. 2015; 143(13):2795-804

3. Karaffova V, Bobikova K, Husakova E, Levkut M, Herich R, Revajova V, Levkutova M, Levkut M. Interaction of TGF-beta4 and IL-17 with IgA secretion in the intestine of chickens fed with E. faecium AL41 and challenged with S. Enteritidis. Res Vet Sci. 2015;(100):75-9.

4. Gomez TM, Motarjemi Y, Miyagawa S, Kaferstein FK, Stohr K. Foodborne salmonellosis. World Health Stat Q. 1997;50(1-2):81-9.

5. Foley SL, Lynne AM. Food animal-associated Salmonella challenges: pathogenicity and antimicrobial resistance. J Anim Sci. 2008;86(14 Suppl): E173-87.

6. Bosilevac JM, Guerini MN, Kalchayanand N, Koohmaraie M. Prevalence and characterization of salmonellae in commercial ground beef in the United States. Appl Environ Microbiol. 2009;75(7):1892-900.

7. Berndt A, Wilhelm A, Jugert C, Pieper J, Sachse K, Methner U. Chicken cecum immune response to Salmonella enterica serovars of different levels of invasiveness. Infect Immun. 2007;75(12):5993-6007.

8. Samiullah, Chousalkar KK, Roberts JR, Sexton M, May D, Kiermeier A. Effects of egg shell quality and washing on Salmonella Infantis penetration. Int J Food Microbiol. 2013;165(2):77-83.

9. Guarnieri DJ, DiLeone RJ. MicroRNAs: a new class of gene regulators. Ann Med. 2008;40(3):197-208. 
10. Ebert MS, Sharp PA. Roles for microRNAs in conferring robustness to biological processes. Cell. 2012;149(3):515-24.

11. Yang $C$, Wei $W$. The miRNA expression profile of the uveal melanoma. Sci China Life Sci. 2011;54(4):351-8.

12. Zhou R, O'Hara SP, Chen XM. MicroRNA regulation of innate immune responses in epithelial cells. Cell Mol Immunol. 2011;8(5):371-9.

13. Takeuchi O, Akira S. Pattern recognition receptors and inflammation. CELL. 2010;140(6):805-20.

14. Schulte LN, Eulalio A, Mollenkopf HJ, Reinhardt R, Vogel J. Analysis of the host microRNA response to Salmonella uncovers the control of major cytokines by the let-7 family. EMBO J. 2011;30(10):1977-89.

15. Sharbati S, Sharbati J, Hoeke L, Bohmer M, Einspanier R. Quantification and accurate normalisation of small RNAs through new custom RT-qPCR arrays demonstrates Salmonella-induced microRNAs in human monocytes. BMC Genomics. 2012:13:23.

16. Bao H, Kommadath A, Plastow GS, Tuggle CK, Guan LL, Stothard P. MicroRNA buffering and altered variance of gene expression in response to Salmonella infection. PLoS ONE. 2014;9(4):e94352.

17. Lawless N, Foroushani AB, McCabe MS, O'Farrelly C, Lynn DJ. Next generation sequencing reveals the expression of a unique miRNA profile in response to a gram-positive bacterial infection. PLoS ONE. 2013;8(3):e57543.

18. Rathjen T, Pais H, Sweetman D, Moulton V, Munsterberg A, Dalmay T. High throughput sequencing of microRNAs in chicken somites. Febs Lett. 2009: 583(9):1422-6

19. Wang Y, Brahmakshatriya V, Zhu H, Lupiani B, Reddy SM, Yoon BJ, Gunaratne $\mathrm{PH}, \mathrm{Kim} J \mathrm{H}$, Chen R, Wang J, et al. Identification of differentially expressed miRNAs in chicken lung and trachea with avian influenza virus infection by a deep sequencing approach. BMC Genomics. 2009;10:512.

20. Kinsella RJ, Kahari A, Haider S, Zamora J, Proctor G, Spudich G, Almeida-King J, Staines D, Derwent P, Kerhornou A, et al. Ensembl BioMarts: a hub for data retrieval across taxonomic space. Database (Oxford). 2011;2011:r30.

21. Wu G, Dawson E, Duong A, Haw R, Stein L. ReactomeFIViz: a Cytoscape app for pathway and network-based data analysis. F1000Res. 2014;3:146.

22. Maudet C, Mano M, Eulalio A. MicroRNAs in the interaction between host and bacterial pathogens. FEBS Lett. 2014;588(22):4140-7.

23. Maudet C, Mano M, Sunkavalli U, Sharan M, Giacca M, Forstner KU, Eulalio A. Functional high-throughput screening identifies the miR-15 microRNA family as cellular restriction factors for Salmonella infection. Nat Commun. 2014;5:4718

24. Staedel C, Darfeuille F. MicroRNAs and bacterial infection. Cell Microbiol. 2013;15(9):1496-507.

25. Wu G, Liu L, Qi Y, Sun Y, Yang N, Xu G, Zhou H, Li X. Splenic gene expression profiling in White Leghorn layer inoculated with the Salmonella enterica serovar Enteritidis. Anim Genet. 2015;46(6):617-26.

26. Ghorai A, Ghosh U. miRNA gene counts in chromosomes vary widely in a species and biogenesis of miRNA largely depends on transcription or posttranscriptional processing of coding genes. Front Genet. 2014;5:100.

27. Sevignani C, Calin GA, Nnadi SC, Shimizu M, Davuluri RV, Hyslop T, Demant P, Croce CM, Siracusa LD. MicroRNA genes are frequently located near mouse cancer susceptibility loci. Proc Natl Acad Sci U S A. 2007;104(19):8017-22.

28. Uribe JH, Collado-Romero M, Zaldivar-Lopez S, Arce C, Bautista R, Carvajal A Cirera S, Claros MG, Garrido JJ. Transcriptional analysis of porcine intestinal mucosa infected with Salmonella Typhimurium revealed a massive inflammatory response and disruption of bile acid absorption in ileum. Vet Res. 2016:47:11

29. Wang Y, Brahmakshatriya V, Lupiani B, Reddy SM, Soibam B, Benham AL, Gunaratne P, Liu HC, Trakooljul N, Ing N, et al. Integrated analysis of microRNA expression and mRNA transcriptome in lungs of avian influenza virus infected broilers. BMC Genomics. 2012;13:278.

30. Lim LP, Lau NC, Garrett-Engele P, Grimson A, Schelter JM, Castle J, Bartel DP, Linsley PS, Johnson JM. Microarray analysis shows that some microRNAs downregulate large numbers of target mRNAs. Nat. 2005;433(7027):769-73.

31. Matarese G, La Cava A. The intricate interface between immune system and metabolism. Trends Immunol. 2004;25(4):193-200.

32. Dumortier $\mathrm{O}$, Hinault C, Van Obberghen E. MicroRNAs and metabolism crosstalk in energy homeostasis. Cell Metab. 2013;18(3):312-24.

33. Rottiers $\mathrm{V}$, Naar AM. MicroRNAs in metabolism and metabolic disorders. Nat Rev Mol Cell Biol. 2012;13(4):239-50

34. Johnston CE, Hartley C, Salisbury AM, Wigley P. Immunological changes at point-of-lay increase susceptibility to Salmonella enterica Serovar enteritidis infection in vaccinated chickens. PLoS ONE. 2012;7(10):e48195.
35. Wigley P, Hulme SD, Powers C, Beal RK, Berchieri AJ, Smith A, Barrow P. Infection of the reproductive tract and eggs with Salmonella enterica serovar pullorum in the chicken is associated with suppression of cellular immunity at sexual maturity. Infect Immun. 2005;73(5):2986-90.

36. Adams J. The proteasome: structure, function, and role in the cell. Cancer Treat Rev. 2003:29 (Suppl 1):3-9.

37. Song R, Harding CV. Roles of proteasomes, transporter for antigen presentation (TAP), and beta 2-microglobulin in the processing of bacterial or particulate antigens via an alternate class I MHC processing pathway. J Immunol. 1996;156(11):4182-90.

38. Kruger E, Kuckelkorn U, Sijts A, Kloetzel PM. The components of the proteasome system and their role in MHC class I antigen processing. Rev Physiol Biochem Pharmacol. 2003;148:81-104.

39. Lo WF, Ong H, Metcalf ES, Soloski MJ. T cell responses to Gram-negative intracellular bacterial pathogens: a role for CD8+ T cells in immunity to Salmonella infection and the involvement of MHC class lb molecules. J Immunol. 1999:162(9):5398-406.

40. Kubori T, Galan JE. Temporal regulation of salmonella virulence effector function by proteasome-dependent protein degradation. Cell. 2003;1 15(3):333-42.

41. Maksymowych WP, Ikawa T, Yamaguchi A, Ikeda M, McDonald D, Laouar L, Lahesmaa R, Tamura N, Khuong A, Yu DT, et al. Invasion by Salmonella typhimurium induces increased expression of the LMP, MECL, and PA28 proteasome genes and changes in the peptide repertoire of HLA-B27. Infect Immun. 1998;66(10):4624-32.

42. Christoffersen NR, Shalgi R, Frankel LB, Leucci E, Lees M, Klausen M, Pilpel Y, Nielsen FC, Oren M, Lund AH. p53-independent upregulation of miR-34a during oncogene-induced senescence represses MYC. Cell Death Differ. 2010;17(2):236-45

43. Li N, Muthusamy S, Liang R, Sarojini H, Wang E. Increased expression of miR-34a and miR-93 in rat liver during aging, and their impact on the expression of Mgst1 and Sirt1. Mech Ageing Dev. 2011;132(3):75-85.

44. Hu H, Wang B, Borde M, Nardone J, Maika S, Allred L, Tucker PW, Rao A. Foxp1 is an essential transcriptional regulator of $B$ cell development. Nat Immunol. 2006;7(8):819-26.

45. Shi C, Zhang X, Chen Z, Sulaiman K, Feinberg MW, Ballantyne CM, Jain MK, Simon DI. Integrin engagement regulates monocyte differentiation through the forkhead transcription factor Foxp1. J Clin Invest. 2004;114(3):408-18.

46. Shi C, Sakuma M, Mooroka T, Liscoe A, Gao H, Croce KJ, Sharma A, Kaplan D, Greaves DR, Wang Y, et al. Down-regulation of the forkhead transcription factor Foxp1 is required for monocyte differentiation and macrophage function. Blood. 2008;112(12):4699-711.

47. Bommer GT, Gerin I, Feng Y, Kaczorowski AJ, Kuick R, Love RE, Zhai Y, Giordano TJ, Qin ZS, Moore BB, et al. p53-mediated activation of miRNA34 candidate tumor-suppressor genes. Curr Biol. 2007;17(15):1298-307.

48. Rao DS, O'Connell RM, Chaudhuri AA, Garcia-Flores Y, Geiger TL, Baltimore D. MicroRNA-34a perturbs B lymphocyte development by repressing the forkhead box transcription factor Foxp1. Immun. 2010:33(1):48-59.

49. Ciraci C, Tuggle CK, Wannemuehler MJ, Nettleton D, Lamont SJ. Unique genome-wide transcriptome profiles of chicken macrophages exposed to Salmonella-derived endotoxin. BMC Genomics. 2010;11:545.

50. Ma L, Cantley LC, Janmey PA, Kirschner MW. Corequirement of specific phosphoinositides and small GTP-binding protein Cdc42 in inducing actin assembly in Xenopus egg extracts. J Cell Biol. 1998;140(5):1125-36.

51. Ma L, Rohatgi R, Kirschner MW. The Arp2/3 complex mediates actin polymerization induced by the small GTP-binding protein $\mathrm{Cdc} 42$. Proc Natl Acad Sci U S A. 1998;95(26):15362-7.

52. Rohatgi R, Ma L, Miki H, Lopez M, Kirchhausen T, Takenawa T, Kirschner MW. The interaction between N-WASP and the Arp2/3 complex links Cdc42dependent signals to actin assembly. Cell. 1999;97(2):221-31.

53. Stender S, Friebel A, Linder S, Rohde M, Mirold S, Hardt WD. Identification of SopE2 from Salmonella typhimurium, a conserved guanine nucleotide exchange factor for Cdc42 of the host cell. Mol Microbiol. 2000;36(6):1206-21.

54. Bhattacharyya S, Borthakur A, Pant N, Dudeja PK, Tobacman JK. BCl10 mediates LPS-induced activation of NF-kappaB and IL-8 in human intestinal epithelial cells. Am J Physiol Gastrointest Liver Physiol. 2007;293(2):G429-37.

55. Ruan WK, Wu YH, An J, Zheng SJ. Polymorphisms of chicken Toll-like receptors 4, 15, and 21 in different breeds. Poult Sci. 2012;91(10):2512-6.

56. MacKinnon KM, He H, Nerren JR, Swaggerty CL, Genovese KJ, Kogut MH. Expression profile of toll-like receptors within the gastrointestinal tract of 2day-old Salmonella enteriditis-infected broiler chickens. Vet Microbiol. 2009; 137(3-4):313-9. 
57. Takeda K, Akira S. Toll-like receptors. Curr Protoc Immunol. 2015;109:12-4.

58. Manicassamy S, Pulendran B. Modulation of adaptive immunity with Toll-like receptors. Semin Immunol. 2009;21(4):185-93.

59. Higuchi M, Matsuo A, Shingai M, Shida K, Ishii A, Funami K, Suzuki $Y$, Oshiumi H, Matsumoto M, Seya T. Combinational recognition of bacterial lipoproteins and peptidoglycan by chicken Toll-like receptor 2 subfamily. Dev Comp Immunol. 2008;32(2):147-55.

60. Langmead B, Salzberg SL. Fast gapped-read alignment with Bowtie 2. Nat Methods. 2012;9(4):357-9.

61. An J, Lai J, Lehman ML, Nelson CC. miRDeep*: an integrated application tool for miRNA identification from RNA sequencing data. Nucleic Acids Res. 2013;41(2):727-37.

62. Robinson MD, McCarthy DJ, Smyth GK. edgeR: a Bioconductor package for differential expression analysis of digital gene expression data. Bioinformatics. 2010;26(1):139-40.

63. John B, Enright AJ, Aravin A, Tuschl T, Sander C, Marks DS. Human MicroRNA targets. PLoS Biol. 2004;2(11):e363.

64. Enright AJ, John B, Gaul U, Tuschl T, Sander C, Marks DS. MicroRNA targets in Drosophila. Genome Biol. 2003;5(1):R1.

65. Dennis GJ, Sherman BT, Hosack DA, Yang J, Gao W, Lane HC, Lempicki RA. DAVID: Database for Annotation, Visualization, and Integrated Discovery. Genome Biol. 2003:4(5):3.

66. Huang DW, Sherman BT, Lempicki RA. Systematic and integrative analysis of large gene lists using DAVID bioinformatics resources. Nat Protoc. 2009;4(1):44-57.

67. Huang DW, Sherman BT, Lempicki RA. Bioinformatics enrichment tools: paths toward the comprehensive functional analysis of large gene lists. Nucleic Acids Res. 2009;37(1):1-13.

68. Al-Shahrour F, Diaz-Uriarte R, Dopazo J. FatiGO: a web tool for finding significant associations of Gene Ontology terms with groups of genes. Bioinformatics. 2004:20(4):578-80.

69. Joslyn CA, Mniszewski SM, Fulmer A, Heaton G. The gene ontology categorizer. Bioinformatics. 2004;20 (Suppl 1):i169-77.

70. Smoot ME, Ono K, Ruscheinski J, Wang PL, Ideker T. Cytoscape 2.8: new features for data integration and network visualization. Bioinformatics. 2011; 27(3):431-2.

\section{Submit your next manuscript to BioMed Central and we will help you at every step:}

- We accept pre-submission inquiries

- Our selector tool helps you to find the most relevant journal

- We provide round the clock customer support

- Convenient online submission

- Thorough peer review

- Inclusion in PubMed and all major indexing services

- Maximum visibility for your research

Submit your manuscript at www.biomedcentral.com/submit 